\title{
Identification of a Gypsy SHOX mutation (p.A170P) in Léri-Weill dyschondrosteosis and Langer mesomelic dysplasia
}

\author{
Verónica Barca-Tierno ${ }^{1,2}$, Miriam Aza-Carmona ${ }^{1,2}$, Eva Barroso ${ }^{1,2}$, Damia Heine-Suner ${ }^{3}$, Dimitar Azmanov ${ }^{4}$, \\ Jordi Rosell $^{3}$, Begoña Ezquieta ${ }^{2,5}$, Lucia Sentchordi Montané ${ }^{6}$, Teresa Vendrell ${ }^{7}$, Jaime Cruz $^{8}$, \\ Fernando Santos ${ }^{1,2}$, José Ignacio Rodríguez ${ }^{9}$, Jesús Pozo ${ }^{10,11,12}$, Jesús Argente ${ }^{10,11,12}$, Luba Kalaydjieva ${ }^{4}$, \\ Ricardo Gracía ${ }^{13}$, Ángel Campos-Barros ${ }^{1,2}$, Sara Benito-Sanz ${ }^{1,2,14}$ and Karen E Heath ${ }^{\star, 1,2,14}$
}

We report the clinical and molecular characteristics of 12 Spanish families with multiple members affected with Léri-Weill dyschondrosteosis (LWD) or Langer mesomelic dysplasia (LMD), who present the SHOX (short stature homeobox gene) mutation p.A170P (c.508G $>$ C) in heterozygosity or homozygosity, respectively. In all studied families, the A170P mutation co-segregated with the fully penetrant phenotype of mesomelic limb shortening and Madelung deformity. A shared haplotype around SHOX was observed by microsatellite analysis, confirming the presence of a common ancestor, probably of Gypsy origin, as 11 of the families were of this ethnic group. Mutation screening in 359 Eastern-European Gypsies failed to identify any carriers. For the first time, we have shown SHOX expression in the human growth plate of a 22-week LMD fetus, homozygous for the A170P mutation. Although the mutant SHOX protein was expressed in all zones of the growth plate, the chondrocyte columns in the proliferative zone were disorganized with the chondrocytes occurring in smaller columnal clusters. We have also identified a novel mutation at the same residue, c. 509C >A (p.A170D), in two unrelated Spanish LWD families, which similar to A170P mutation impedes nuclear localization of SHOX. In conclusion, we have identified A170P as the first frequent SHOX mutation in Gypsy LWD and LMD individuals.

European Journal of Human Genetics (2011) 19, 1218-1225; doi:10.1038/ejhg.2011.128; published online 29 June 2011

Keywords: SHOX; A170P; A170D; Léri-Weill dyschondrosteosis; Langer mesomelic dysplasia; founder

\section{INTRODUCTION}

Léri-Weill dyschondrosteosis (LWD; MIM 127300) is a dominantly inherited skeletal dysplasia characterized by disproportionate short stature, mesomelic limb shortening and the Madelung deformity: bowing of the radius and distal dislocation of the ulna. ${ }^{1}$ Langer mesomelic dysplasia (LMD; MIM 249700) represents a more severe clinical form with disproportionate short stature due to shortening of the mesomelic and rhizomelic limb segments, abnormality of the humeral head, angulation of the radial shaft, carpal distortion, short femoral neck and hypoplastic or absent proximal half of the fibula. ${ }^{2}$

SHOX (short stature homeobox gene, MIM 312865), localized within the pseudoautosomal region 1 (PAR1) of the $\mathrm{X}$ and $\mathrm{Y}$ chromosomes ${ }^{3,4}$ encodes a transcription factor implicated in skeletal growth. ${ }^{5}$ SHOX haploinsufficiency results in LWD, whereas total deficiency results in LMD. ${ }^{6-8}$ Mutations include deletions of $S H O X^{9,10}$ or the downstream enhancer region, ${ }^{11-14}$ partial or complete
SHOX duplications, ${ }^{15}$ or point or insertion/deletion mutations within SHOX.

The SHOX missense mutation p.A170P was originally identified in a large consanguineous Spanish Gypsy LWD/LMD family, ${ }^{16}$ and subsequently reported by us in a second Spanish Gypsy family. ${ }^{17}$ Alanine 170 is localized within the five amino acids of the homeodomain, which form the SHOX nuclear localization signal. ${ }^{16,18}$ Functional studies showed that the mutant protein was unable to enter the nucleus, thus impairing its function as a transcription factor. ${ }^{16}$

During routine analysis of $\mathrm{LWD} / \mathrm{LMD}$ referrals, we have identified a total of 12 Spanish families presenting with the p.A170P mutation, including the two previously described, ${ }^{16,17}$ and two Spanish families with a previously undescribed mutation at the same residue, p.A170D. Functional analysis of the A170D mutant showed that, similar to A170P, nuclear translocation was impaired. We subsequently

\footnotetext{
${ }^{1}$ Instituto de Genética Médica y Molecular, Hospital Universitario La Paz, Universidad Autónoma de Madrid, Madrid, Spain; ${ }^{2}$ Centro de Investigación Biomédica en Red de Enfermedades Raras, Instituto de Salud Carlos III, Madrid, Spain; ${ }^{3}$ Department of Genetics, Hospital Son Espases, Palma de Mallorca, Spain; ${ }^{4}$ Laboratory of Molecular Genetics, Western Australian Institute for Medical Research, UWA Centre for Medical Research, University of Western Australia, Perth, Australia; ${ }^{5}$ Department of Biochemistry, Hospital Universitario Gregorio Marañón, Madrid, Spain; ${ }^{6}$ Department of Pediatrics, Hospital Infanta Leonor, Madrid, Spain; ${ }^{7}$ Department of Genetics, Hospital Universitario Vall d' Hebron, Universidad Autónoma de Barcelona, Barcelona, Spain; ${ }^{8}$ Department of Pediatric Endocrinology, Hospital Universitario 12 de Octubre, Madrid, Spain; ${ }^{9}$ Department of Pathology, Hospital Universitario La Paz, Madrid, Spain; ${ }^{10}$ Department of Endocrinology, Hospital Infantil Universitario Niño Jesús, Instituto de Investigación La Princesa, Madrid, Spain; ${ }^{11}$ Department of Pediatrics, Universidad Autónoma de Madrid, Madrid, Spain; ${ }^{12}$ Centro de Investigación Biomédica en Red de Fisiopatología, Obesidad y Nutrición (CIBEROBN), Instituto de Salud Carlos III, Madrid, Spain; ${ }^{13}$ Department of Pediatric Endocrinology, Hospital Universitario La Paz, Universidad Autónoma de Madrid, Madrid, Spain

*Correspondence: Dr KE Heath, Instituto de Genética Médica y Molecular, Hospital Universitario La Paz, Universidad Autónoma de Madrid, P Castellana 261, Madrid 28046, Spain. Tel: +34 91727 7469; Fax: +34 91207 1040; E-mail: kheath.hulp@salud.madrid.org

${ }^{14}$ These authors contributed equally to this work.

Received 9 March 2011; revised 26 May 2011; accepted 1 June 2011; published online 29 June 2011
} 
undertook haplotype analysis around SHOX in these families to investigate the possibility of common ancestry for each mutation or whether alanine 170 represents a mutation hotspot. A common haplotype around SHOX was observed for the 12 families with the A170P mutation suggesting a common ancestor, whereas no haplotype was shared between the two A170D individuals.

We had the opportunity to evaluate SHOX expression in the human growth plate of a 22-week LMD fetus, confirmed to be an A170P homozygote. The chondrocytes in the reserve zone appeared enlarged and in pairs, whereas the columns in the proliferative zone were disorganized.

In summary, we report the first founder SHOX mutation, A170P, in the Gypsy LWD population and the presence of another mutation at the same residue, A170D, in two LWD families.

\section{SUBJECTS AND METHODS}

\section{Subjects}

The study was approved by the local ethics committees from the various institutions. Informed consent for the study was provided by all the participants or their legal representatives. Genomic DNA was isolated from whole blood, amniotic fluid or paraffin-embedded tissue using the Blood core B kit (Qiagen, Valencia, CA, USA), Master Pure DNA purification kit (Epicentre, Illumina, Madison, WI, USA) or QIAamp DNA FFPE tissue kit (Qiagen), respectively. The pedigrees of the $12 \mathrm{~A} 170 \mathrm{P}$ families are shown in Figure 1, whereas the clinical data is listed in Table 1. The height range of LWD and LMD A170P affected individuals was -1.7 to $-5.6 \mathrm{SD}$ and -8.2 to $-10.2 \mathrm{SD}$, respectively. The transabdominal ultrasonagraphical data and the post-termination radiographs of the LMD fetus (family 11, IV.9) are shown in Supplementary Figure 1.

Three control cohorts were analyzed for the mutation or for the associated haplotype: (1) 88 'healthy unrelated' Spanish Gypsies, (2) 359 EasternEuropean Roma individuals: 233 of whom belonged to the Balkan and 126 to the Vlax migrational category and (3) 173 Spanish control subjects with heights within the normal range for the Spanish population for age and gender $(-2<$ SDS $<+2)$, obtained from the Spanish DNA bank (University of Salamanca, Spain)

\section{Mutation detection}

Deletion and duplication analysis was carried out using the commercial SHOX/ PAR1 MLPA Kit (MRC Holland, Amsterdam, The Netherlands) and in accordance to the manufacturer's recommendations. SHOX mutations were screened using various techniques: high-resolution melting analysis (HRM) in a LightScanner HR96 system (Idaho Technologies, Salt Lake City, UT, USA), dHPLC (WAVE 4500HT, Transgenomic, Omaha, MO, USA) and/or DNA sequencing (ABI 3130, Applied Biosystems, Foster City, CA, USA), as previously described. ${ }^{17}$

The A170P mutation was screened in the Eastern-European Roma population using HRM on the Lightcycler 480 (Roche Applied Bioscience, Roche, Mannheim, Germany). The PCR conditions were $1 \times$ Roche Lightcycler master mix, $3.0 \mathrm{mM} \mathrm{MgCl}_{2}, 0.2 \mu \mathrm{m}$ of each oligonucleotide, 5\% DMSO and $10 \mathrm{ng}$ DNA

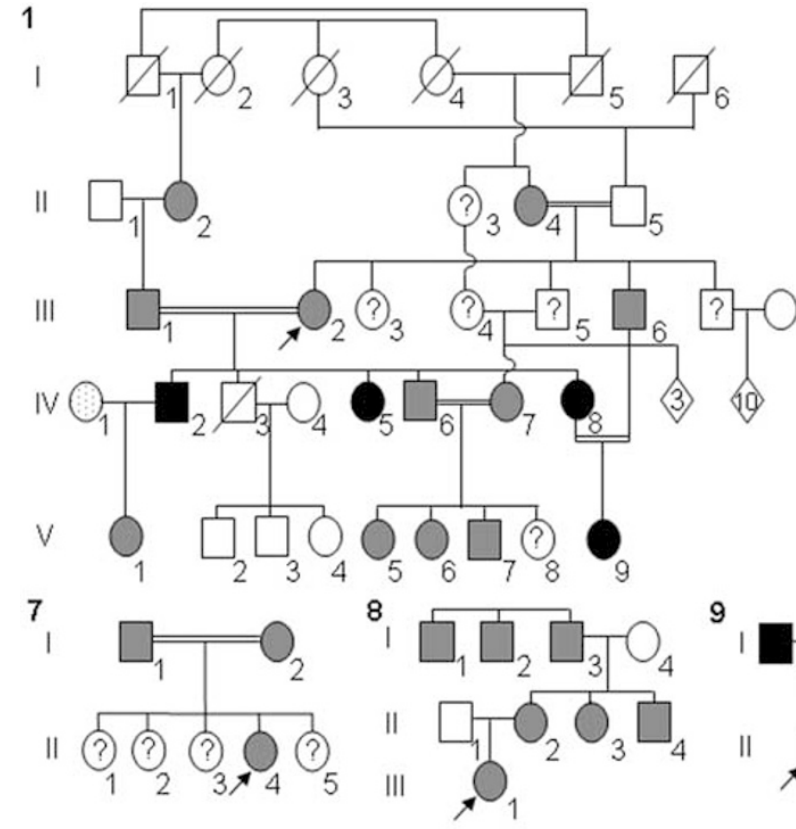

11

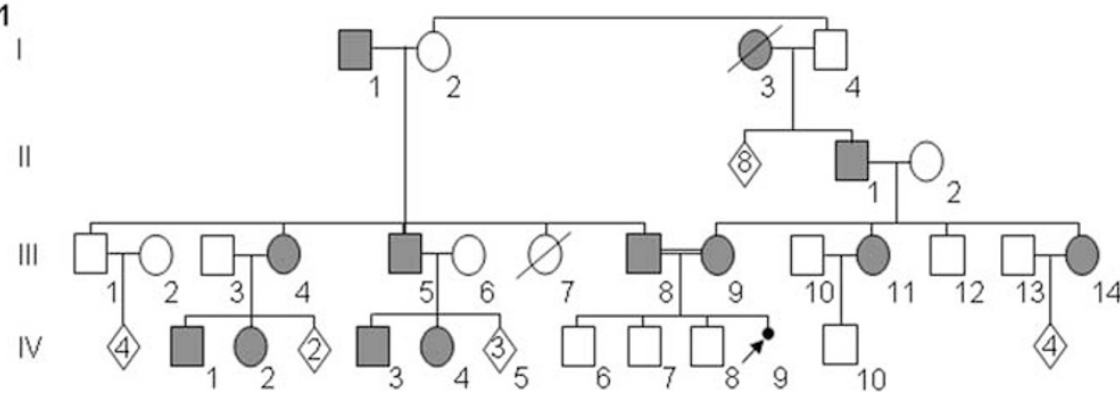

II

III
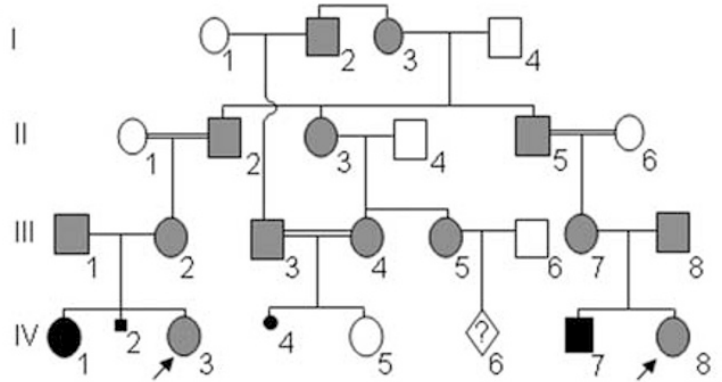

3

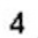

5
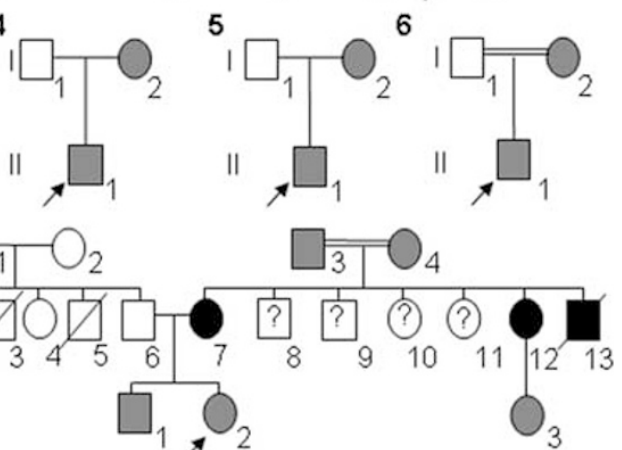

12

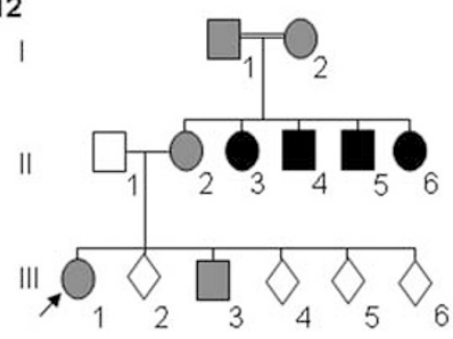

Figure 1 Pedigrees of the 12 LWD/LMD families carrying the A170P SHOX mutation. Individuals with LWD are shown as gray filled symbols, whereas individuals with LMD are shown as black filled symbols. Individual IV.1 of family 1 was clinically and genetically diagnosed with achondroplasia (FGFR3 c.1138G >A, G380R). Unknown diagnosis is shown by a question marker. 
Table 1 Clinical details of the documented LWD and LMD affected individuals and the prenatal diagnosis undertaken in the 12 Spanish families

\begin{tabular}{|c|c|c|c|c|c|c|c|c|c|c|}
\hline Family & $\begin{array}{l}\text { Ethnic } \\
\text { origin }\end{array}$ & $\begin{array}{c}\text { Pedigree } \\
\text { individual No. }\end{array}$ & $\begin{array}{l}\text { Sex } \\
(F / M)\end{array}$ & Status & $\begin{array}{l}\text { Gen. } \\
\text { Conf. }\end{array}$ & $\begin{array}{c}\text { Age } \\
\text { (years) }\end{array}$ & $\begin{array}{l}\text { Height } \\
(\mathrm{cm})\end{array}$ & $\begin{array}{l}\text { Height } \\
\text { SDS }\end{array}$ & $M D$ & $\begin{array}{l}\text { Other clinical and } \\
\text { auxological details }\end{array}$ \\
\hline 1 & Gypsy & 11.4 & $\mathrm{~F}$ & LWD & - & Adult & 140.0 & -3.7 & MD & - \\
\hline 1 & Gypsy & III.2 & $\mathrm{F}$ & LWD & $Y$ & Adult & 140.8 & -3.6 & MD & - \\
\hline 1 & Gypsy & III.6 & $M$ & LWD & - & Adult & 148.0 & -4.5 & MD & - \\
\hline 1 & Gypsy & IV. 2 & $M$ & LMD & $Y$ & Adult & SS & - & MD & - \\
\hline 1 & Gypsy & IV.5 & $F$ & LMD & - & Adult & 109.0 & -9.1 & - & - \\
\hline 1 & Gypsy & IV.6 & $M$ & LWD & $Y$ & Adult & SS & - & MD & - \\
\hline 1 & Gypsy & IV.7 & $F$ & LWD & $Y$ & Adult & SS & - & MD & - \\
\hline 1 & Gypsy & IV.8 & $\mathrm{F}$ & LMD & - & Adult & 110.0 & -9.0 & - & - \\
\hline 1 & Gypsy & V.1 & $\mathrm{F}$ & LWD & $Y$ & Adult & SS & - & MD & - \\
\hline 1 & Gypsy & V.5 & $\mathrm{F}$ & LWD & $Y$ & Adult & SS & - & MD & - \\
\hline 1 & Gypsy & V.6 & $\mathrm{F}$ & LWD & $Y$ & Adult & SS & - & MD & - \\
\hline 1 & Gypsy & V.7 & M & LWD & $Y$ & 12.0 & 116.6 & -5.1 & MD & - \\
\hline 1 & Gypsy & V.9 & $\mathrm{F}$ & LMD & - & 7.0 & 89.0 & -8.0 & - & - \\
\hline 2 & Gypsy & III.1 & $M$ & LWD & $Y$ & Adult & SS & - & MD & - \\
\hline 2 & Gypsy & III.2 & $F$ & LWD & - & Adult & SS & - & MD & - \\
\hline 2 & Gypsy & III.3 & $M$ & LWD & $Y$ & Adult & SS & - & MD & - \\
\hline 2 & Gypsy & III.4 & $\mathrm{F}$ & LWD & $Y$ & Adult & SS & - & MD & - \\
\hline 2 & Gypsy & III.5 & $\mathrm{F}$ & LWD & $Y$ & Adult & SS & - & $\mathrm{MD}$ & - \\
\hline 2 & Gypsy & III.7 & $\mathrm{F}$ & LWD & $Y$ & Adult & SS & - & MD & - \\
\hline 2 & Gypsy & III.8 & $M$ & LWD & $Y$ & Adult & SS & - & MD & - \\
\hline 2 & Gypsy & IV.1 & $F$ & LMD & - & Fetus & SS & - & - & - \\
\hline 2 & Gypsy & IV.2 & $M$ & LMD & - & Fetus & $N A$ & NA & $N A$ & $\begin{array}{l}\text { Electively aborted at } 16 \text { weeks gestation due to } \\
\text { severe limb shortening with ultrasonography } \\
\text { No genetic analysis undertaken }\end{array}$ \\
\hline $2^{a}$ & Gypsy & IV.3 & $\mathrm{F}$ & LWD & $Y$ & Fetus & $N A$ & NA & NA & Prenatal diagnosis at 19 weeks gestation \\
\hline $2^{\mathrm{a}}$ & Gypsy & IV.4 & $\mathrm{F}$ & LMD & Y & Fetus & NA & NA & NA & Electively aborted after prenatal diagnosis at 18 weeks gestation \\
\hline 2 & Gypsy & IV.5 & $\mathrm{F}$ & $\mathrm{N}$ & Y & Fetus & NA & NA & NA & Prenatal diagnosis at 16 weeks gestation \\
\hline 2 & Gypsy & IV.6 & ND & ND & ND & Fetus & NA & NA & NA & No prenatal diagnosis undertaken as risk of LWD but not LMD \\
\hline 2 & Gypsy & IV.7 & M & LMD & - & Child & SS & - & - & \\
\hline $2^{\mathrm{a}}$ & Gypsy & IV.8 & $\mathrm{F}$ & LWD & Y & Fetus & NA & NA & NA & Prenatal diagnosis at 19 weeks gestation \\
\hline 3 & Gypsy & 1.2 & $\mathrm{~F}$ & LWD & Y & Adult & SS & - & MD & - \\
\hline 3 & Gypsy & II. 1 & M & LWD & Y & 10.0 & SS & - & MD & - \\
\hline 4 & Spanish & 1.2 & $\mathrm{~F}$ & LWD & Y & Adult & 142.5 & -3.3 & MD & - \\
\hline 4 & Spanish & II.1 & M & LWD & Y & 3.5 & 88.5 & -2.7 & MD & - \\
\hline 5 & Gypsy & 1.2 & $\mathrm{~F}$ & LWD & Y & Adult & 143.0 & -3.2 & MD & - \\
\hline 5 & Gypsy & II.1 & M & LWD & Y & 14.4 & 145.0 & -2.4 & MD & - \\
\hline 6 & Gypsy & 1.2 & $\mathrm{~F}$ & LWD & Y & Adult & 146.1 & -2.6 & MD & Cervical scoliosis \\
\hline 6 & Gypsy & II.1 & M & LWD & Y & 13.4 & 134.1 & -2.8 & MD & Cervical scoliosis \\
\hline 7 & Gypsy & 11.4 & $\mathrm{~F}$ & LWD & Y & 11.8 & 113.0 & -5.6 & MD & Cervical scoliosis \\
\hline 8 & Gypsy & 11.2 & $\mathrm{~F}$ & LWD & Y & Adult & 135.0 & -4.6 & MD & - \\
\hline 8 & Gypsy & III.1 & $\mathrm{F}$ & LWD & Y & 6.5 & 99.5 & -3.7 & MD & - \\
\hline 9 & Gypsy & I.1 & M & LMD & - & Adult & 120 & -8.2 & MD & - \\
\hline 9 & Gypsy & II.1 & $\mathrm{F}$ & LWD & Y & Child & SS & - & MD & - \\
\hline 10 & Gypsy & 11.7 & $\mathrm{~F}$ & LMD & $Y$ & Adult & 101.0 & -10.2 & MD & - \\
\hline 10 & Gypsy & III.1 & $\mathrm{F}$ & LWD & $Y$ & 8.5 & SS & - & MD & - \\
\hline 11 & Gypsy & $\mathrm{I} .1$ & $M$ & LWD & - & Adult & SS & - & MD & - \\
\hline 11 & Gypsy & 1.3 & $F$ & LWD & - & Adult & SS & - & MD & - \\
\hline 11 & Gypsy & 1.9 & $M$ & LWD & - & Adult & 143.0 & -3.7 & MD & - \\
\hline 11 & Gypsy & III.4 & $\mathrm{F}$ & LWD & - & Adult & 145.0 & -3.3 & MD & - \\
\hline 11 & Gypsy & III.5 & $M$ & LWD & - & Adult & 155.0 & -3.6 & MD & - \\
\hline 11 & Gypsy & III.8 & $M$ & LWD & $Y$ & Adult & 152.0 & -4.1 & MD & - \\
\hline 11 & Gypsy & 111.9 & $\mathrm{~F}$ & LWD & $Y$ & Adult & 144.0 & -3.5 & MD & - \\
\hline 11 & Gypsy & III.11 & $\mathrm{F}$ & LWD & - & Adult & 140.0 & -4.2 & MD & - \\
\hline 11 & Gypsy & III.14 & $\mathrm{F}$ & LWD & - & Adult & 147.0 & -3.0 & MD & - \\
\hline 11 & Gypsy & IV. 1 & $M$ & LWD & - & ND & SS & - & ND & - \\
\hline 11 & Gypsy & IV. 2 & $\mathrm{~F}$ & LWD & - & ND & SS & - & ND & - \\
\hline 11 & Gypsy & IV.3 & $M$ & LWD & - & ND & SS & - & ND & - \\
\hline 11 & Gypsy & IV. 4 & $\mathrm{~F}$ & LWD & - & ND & SS & - & ND & - \\
\hline
\end{tabular}


Table 1 (Continued)

\begin{tabular}{|c|c|c|c|c|c|c|c|c|c|c|}
\hline Family & $\begin{array}{l}\text { Ethnic } \\
\text { origin }\end{array}$ & $\begin{array}{c}\text { Pedigree } \\
\text { individual No. }\end{array}$ & $\begin{array}{l}\text { Sex } \\
(F / M)\end{array}$ & Status & $\begin{array}{l}\text { Gen. } \\
\text { Conf. }\end{array}$ & $\begin{array}{l}\text { Age } \\
\text { (years) }\end{array}$ & $\begin{array}{l}\text { Height } \\
(\mathrm{cm})\end{array}$ & $\begin{array}{l}\text { Height } \\
\text { SDS }\end{array}$ & $M D$ & $\begin{array}{l}\text { Other clinical and } \\
\text { auxological details }\end{array}$ \\
\hline $11^{\mathrm{b}}$ & Gypsy & IV.9 & M & LMD & Y & Fetus & SS & $-^{a}$ & MD & $\begin{array}{l}\text { Electively aborted at } 22 \text { weeks gestation after prenatal diagnosis } \\
\text { Body length } 25 \mathrm{~cm} \text {, humerus } 30 \mathrm{~mm} \text {, radius } 19 \mathrm{~mm} \text {, ulna } 9 \mathrm{~mm} \text {, } \\
\text { femur } 32 \mathrm{~mm} \text {, tibia } 25 \mathrm{~mm} \text {, fibia } 11 \mathrm{~mm} \text { (Supplemetary Figure 1) }\end{array}$ \\
\hline 12 & Gypsy & 11.2 & $\mathrm{~F}$ & LWD & Y & Adult & 142.0 & -3.4 & MD & - \\
\hline 12 & Gypsy & III.1 & $\mathrm{F}$ & LWD & Y & 9.6 & 127.0 & -1.7 & MD & - \\
\hline
\end{tabular}

Abbreviations: LMD, Langer mesomelic dysplasia; LWD, Léri-Weill dyschondrosteosis; MD, Madelung deformity; N, normal; NA, no measurements could be undertaken as the individual was a fetus; ND; not determined; SS, short stature.

arenatal analysis undertaken with amniocentesis material.

${ }^{b}$ Analysis undertaken using DNA extracted from aborted tissue.

Status: $L W D$ and $L M D=A 170 P$ mutation in heterozygosity and homozygosity, respectively.

in a total volume of $15 \mu \mathrm{l}$. The sense and antisense oligos were $5^{\prime}$-CT TGGTTCAGCCTCATGGGAAG- $3^{\prime}$ and $5^{\prime}$-TGTGGTGGTCCTGGGTGTAGC C-3', respectively. The PCR cycling and HRM conditions were according to the manufacturer's recommendations (Roche Applied Bioscience).

\section{Haplotype analysis}

Haplotype analysis was undertaken to determine if a common ancestor was present for the two different mutations. Eight microsatellite markers located intragenically or in the $5^{\prime}$ and $3^{\prime}$ flanking regions of SHOX were analyzed as previously described. ${ }^{11,17,19}$ The probability of observing the associated haplotype in the Spanish Gypsy and control populations was determined by analyzing the genotypes for each microsatellite marker in at least 100 chromosomes of the respective populations.

\section{Subcellular localization studies}

The A170D and A170P mutations were introduced into the vector pFLAGCMV-1 (Sigma-Aldrich, St Louis, MO, USA) containing the full-length SHOXa cDNA using primers $5^{\prime}$-TCCAGAACCGGAGACCCAAGTGCCGCAAAC- ${ }^{\prime}$ and 5'-GGTTCCAGAACCGGAGACCCAAGTGCCGCAAACAAG-3', respectively, and the QuikChange II XL Site-Directed Mutagenesis Kit (Stratagene, Agilent Technologies, Santa Clara, CA, USA) according to the manufacturer's recommendations.

Immunocytochemistry: U2OS human osteosarcoma cells (Invitrogen, Life Technologies, Carlsbad, CA, USA) were grown in Dulbecco's modified Eagles medium supplemented with antibiotics and $10 \%$ fetal bovine serum (Invitrogen, Life Technologies) for $24 \mathrm{~h}$ on four-well chamber slides (Becton Dickinson, Franklin Lakes, NJ, USA). Transient transfection of the different plasmid constructs was undertaken using FuGene (Roche Applied Bioscience) according to the manufacturer's instructions. At $24 \mathrm{~h}$ post transfection, the cells were washed and fixed with $4 \%$ paraformaldehyde (Sigma-Aldrich). Briefly, the cells were then washed with PBS and blocked with glycine buffer (1\% BSA, $0.3 \mathrm{M}$ glycine, Tween $0.2 \%$ diluted in PBS (PBS-T)). The preparations were incubated overnight at $4{ }^{\circ} \mathrm{C}$ with a rabbit polyclonal SHOX antibody. ${ }^{20}$ The cells were then washed with PBS-T and incubated with the secondary antibody, Alexa fluor 488 goat anti-rabbit IgG (Commercial Molecular Probes, Invitrogen). The cells were subsequently washed again with PBS-T, counterstained with DAPI (Sigma-Aldrich) and mounted with Mowiol 4-88 (Sigma-Aldrich). SHOX and DAPI localization were visualized using a confocal microscope SP5 (Leica, Leica Microsystems GmbH, Wetzlar, Germany).

Immunohistochemistry: Human radius and ulna growth plate sections were obtained from an electively aborted 22-week LMD fetus, confirmed to be an A170P homozygote by prenatal analysis (family 11, individual IV.9) and a spontaneously aborted 'healthy' 23 -week fetus. Parents had provided informed consent for the use of their fetus for scientific research relevant to the clinical diagnosis. The LMD fetus was confirmed to carry two copies of the A170P mutation. Subsequently, histological analysis was undertaken as previously described. ${ }^{20}$ Controls for specific antibody staining were performed by (1) using preimmune serum from the rabbit immunized against the SHOX peptide, at a dilution of 1:1000; (2) replacing the primary antibody with PBS; (3) using a rabbit polyclonal IgG isotype control (Ab27472, Abcam, Cambridge, UK) at a dilution of 1:50 and (4) staining sections of a normal adult colon with the SHOX antibody. Sections were examined using a DM5500B fluorescent microscope (Leica), and photos were taken using a DFC360FX digital camera (Leica) and visualized using the LA software (Leica).

\section{RESULTS}

\section{Mutation detection and haplotype analysis}

The SHOX mutation p. A170P (c.508G >C) was identified in 12 LWD/LMD probands (Figure 1). In the 12 families, a total of 34 LWD and four LMD individuals were subsequently genetically analyzed, all of whom were heterozygous or homozygous for the mutation, respectively.

The analysis of SHOX intragenic and flanking microsatellite markers revealed a common haplotype, present in one or two copies in all LWD and LMD affected family members, respectively. The haplotype block extended at least $77 \mathrm{~kb}$, from microsatellite marker DXYS10038 to DXYS10093, to a maximum length of $165 \mathrm{~kb}$ (Table 2). Multiple haplotypes were observed with increasing physical distance from SHOX, suggesting the occurrence of multiple recombination events (Table 2). The frequency of this haplotype was determined in two control cohorts; 1 in 40062 in Spanish Gypsies and 1 in 390000 Spanish normal height controls.

As the A170P mutation was identified in 11 Spanish Gypsy families, we investigated its history. We looked into the genealogy of each family by last names and family interviews. Interestingly, the data obtained from one of the homozygous Gypsy probands (family 10) suggested that her family had migrated to Spain several generations ago from Eastern Europe. The common haplotype was observed in this family in the affected LWD and LMD individuals. Subsequently, we screened a panel of population controls from Bulgaria, representing Gypsy groups that remained in the Balkans during the early Gypsy diaspora across Europe. No A170P mutation was identified.

In two additional Spanish non-Gypsy families, we identified a novel missense mutation at the same residue, A170D (c.509C>A). No common SHOX haplotype was identified (data not shown).

\section{Subcellular localization}

We introduced the A170P and A170D missense mutations into the full-length SHOX cDNA by site-directed mutagenesis. The wild-type and mutant plasmids were transiently transfected into U2OS osteosarcoma cells, and the subcellular localization of the different SHOX proteins was analyzed by immunocytochemistry. Both A170D and A170P mutant proteins failed to localize to the nucleus, showing that nuclear translocation was impaired (Figure 2).

\section{SHOX expression in a LMD human growth plate}

We compared the expression of mutant SHOX in the human growth plate of a 22-week old fetus, molecularly confirmed to be homozygous 
Table 2 Haplotypes determined from SHOX intragenic or flanking microsatellite markers in the 12 LWD/LMD families

\begin{tabular}{|c|c|c|c|c|c|c|c|c|c|c|c|c|c|}
\hline Family & 1 & 1 & 1 & 1 & 1 & 1 & 1 & 1 & 1 & 1 & 2 & 2 & 2 \\
\hline Member & III.2 & III.6 & IV.1 & IV.2 & IV6 & IV.7 & V.1 & V. 5 & V. 6 & V.7 & III.1 & III.2 & III.3 \\
\hline Status & LWD & LWD & $\mathrm{ACH}$ & LMD & LWD & LWD & LWD & LWD & LWD & LWD & LWD & LWD & LWD \\
\hline DXYS10037 & $180-180$ & $180-180$ & $200-180$ & $180-180$ & $180-180$ & $200-180$ & $180-180$ & $180-180$ & 180-200 & $180-180$ & 180-198 & $180-200$ & $200-180$ \\
\hline DXYS10038 & $217-217$ & $217-217$ & $209-213$ & $217-217$ & $217-217$ & $217-217$ & 213-217 & $217-217$ & 217-217 & 217-217 & 217-217 & 217-213 & 209-217 \\
\hline DXYS201 & 135-155 & 135-155 & $145-155$ & $155-155$ & 135-155 & 157-155 & 155-155 & 135-155 & $155-157$ & 135-155 & $155-135$ & $155-147$ & 141-155 \\
\hline DXYS10092 & $357-359$ & $357-359$ & $363-363$ & $359-359$ & $357-359$ & $347-359$ & $363-359$ & $357-359$ & $359-347$ & $357-359$ & $359-345$ & $359-361$ & $341-359$ \\
\hline DYS290 & 292-292 & 292-292 & $306-296$ & $292-292$ & 292-292 & 296-292 & 296-292 & 292-292 & 292-296 & 292-292 & 292-292 & 292-292 & 294-292 \\
\hline DXYS10093 & 249-235 & $247-235$ & $253-247$ & $235-235$ & $247-235$ & $235-235$ & $247-235$ & $247-235$ & 235-235 & $247-235$ & 235-251 & $235-245$ & 251-235 \\
\hline DXYS10083 & $156-160$ & $156-160$ & $170-160$ & $160-160$ & $156-160$ & $154-160$ & $160-160$ & $156-160$ & $160-154$ & $156-160$ & $160-172$ & $160-154$ & $160-162$ \\
\hline DXYS10085 & $218-224$ & $218-224$ & $226-210$ & $224-224$ & $218-224$ & $222-224$ & $210-224$ & $218-224$ & 224-222 & $218-224$ & $216-224$ & $216-220$ & $216-222$ \\
\hline Family & 2 & 2 & 2 & 2 & 2 & 2 & 2 & 2 & 2 & 3 & 3 & 4 & 4 \\
\hline Member & III.4 & III. 5 & III.6 & III.7 & III.8 & IV.3 & IV. 4 & IV.5 & IV. 8 & 1.2 & II.1 & 1.2 & II.1 \\
\hline Status & LWD & LWD & $\mathrm{N}$ & LWD & LWD & LWD & LMD & $N$ & LWD & LWD & LWD & LWD & LWD \\
\hline DXYS10037 & $200-180$ & $200-180$ & $196 \sim 200$ & $200-180$ & 180-200 & $198-180$ & $180-180$ & $200-200$ & 180-200 & 200-180 & $202-180$ & $202-180$ & $202-180$ \\
\hline DXYS10038 & 209-217 & 209-217 & $197 \sim 197$ & 217-197 & 209-217 & $217-217$ & $217-217$ & 209-209 & 197-217 & 209-217 & 213-217 & 209-217 & 209-217 \\
\hline DXYS201 & 143-155 & 143-155 & $149 \sim 155$ & $155-151$ & 159-155 & 135-155 & $155-155$ & $151-143$ & $151 \sim 155$ & 143-155 & 151-155 & 143-155 & 143-155 \\
\hline DXYS10092 & $341-359$ & $341-359$ & $347 \sim 355$ & $359-343$ & 349-359 & 345-359 & $359-359$ & $341-341$ & 343-359 & 359-359 & $343-359$ & $347-359$ & $347-359$ \\
\hline DYS290 & $306-292$ & 306-292 & $296 \sim 306$ & $292-306$ & 292-292 & 292-292 & 292-292 & 294-306 & 306-292 & 296-292 & 296-292 & 296-292 & 296-292 \\
\hline DXYS10093 & $245-235$ & $245-235$ & $235 \sim 255$ & $235-245$ & 249-235 & 251-235 & $235-235$ & $251-245$ & $245-235$ & 243-235 & 235-235 & 261-235 & $261-235$ \\
\hline DXYS10083 & $160-160$ & $160-160$ & $160 \sim 162$ & $160-160$ & $170-160$ & $172-160$ & $162-160$ & $162-160$ & $160-160$ & $158-160$ & $154-160$ & $158-160$ & $154-160$ \\
\hline DXYS10085 & $214-216$ & $214-216$ & $220 \sim 222$ & 216-214 & 216-212 & $224-216$ & $222-216$ & $222-214$ & 214-212 & 218-214 & $224-214$ & 218-214 & 224-214 \\
\hline Family & 5 & 5 & 5 & 6 & 6 & 6 & 7 & 8 & 8 & 8 & 9 & 10 & 10 \\
\hline Member & I.1 & 1.2 & II. 1 & I. 1 & 1.2 & II.1 & 11.4 & II. 1 & II.2 & III.1 & II.1 & II.6 & 11.7 \\
\hline Status & $\mathrm{N}$ & LWD & LWD & $\mathrm{N}$ & LWD & LWD & LWD & $\mathrm{N}$ & LWD & LWD & LWD & $\mathrm{N}$ & LMD \\
\hline DXYS10037 & 200-192 & $200-180$ & $192-180$ & 194-200 & 198-180 & 200-180 & 180-180 & 198-200 & 180-190 & 200-180 & 180-198 & 198-192 & $180-180$ \\
\hline DXYS10038 & 209-213 & 209-217 & 213-217 & 209-209 & 199-217 & 209-217 & 217-217 & 213-217 & 217-217 & 217-217 & 213-217 & $197-217$ & 217-217 \\
\hline DXYS201 & $143-149$ & $143-155$ & 149-155 & $143-159$ & 149-155 & 159-155 & $143-155$ & $145-151$ & $155-145$ & 151-155 & $147-155$ & $135-145$ & $155-155$ \\
\hline DXYS10092 & $355-343$ & $355-359$ & 343-359 & $347-349$ & 349-359 & 349-359 & 339-359 & $361-353$ & $359-361$ & 353-359 & $361-359$ & $317-361$ & 359-359 \\
\hline DYS290 & 296-296 & 296-292 & 296-292 & 292-296 & 296-292 & 296-292 & 296-292 & 296-292 & 292-306 & 292-292 & 292-292 & 292-306 & 292-292 \\
\hline DXYS10093 & $235-235$ & $235-235$ & $235-235$ & $233-233$ & $247-235$ & $233-235$ & $253-235$ & $235-231$ & $235-257$ & $231-235$ & $245-235$ & $235-257$ & $235-235$ \\
\hline DXYS10083 & $162-154$ & $154-160$ & $154-160$ & $154-154$ & $156-160$ & $154-160$ & $162-160$ & $156-158$ & $162-160$ & $158-160$ & $164-160$ & $160-162$ & $160-160$ \\
\hline DXYS10085 & $216-224$ & $216-214$ & $224-214$ & $220-224$ & $222-214$ & 224-214 & $216-214$ & $224-228$ & 216-212 & 228-216 & $224-216$ & $222-212$ & 224-224 \\
\hline Family & 10 & 11 & 11 & 11 & 12 & 12 & 12 & & & & & & \\
\hline Member & III.2 & III.8 & IV.9 & III.9 & II. 1 & II.2 & III.1 & & & & & & \\
\hline Status & LWD & LWD & LMD & LWD & N & LWD & LWD & & & & & & \\
\hline DXYS10037 & $192-180$ & $180-180$ & $180-180$ & $202-180$ & $202-180$ & $196-180$ & $180-180$ & & & & & & \\
\hline DXYS10038 & 217-217 & 209-217 & 217-217 & 209-217 & 209-217 & 213-217 & 217-217 & & & & & & \\
\hline DXYS201 & $145-155$ & 145-155 & $155-155$ & 143-155 & $151-137$ & 145-155 & 137-155 & & & & & & \\
\hline DXYS10092 & $361-359$ & $387-359$ & 359-359 & 345-359 & $353-357$ & $357-359$ & $357-359$ & & & & & & \\
\hline DYS290 & $306-292$ & 296-292 & 292-292 & $308-292$ & 292-296 & 292-292 & 296-292 & & & & & & \\
\hline DXYS10093 & $257-235$ & $243-235$ & $235-235$ & $243-235$ & $251-233$ & $251-235$ & $233-235$ & & & & & & \\
\hline DXYS10083 & $162-160$ & $164-154$ & $154-160$ & $154-160$ & $158-158$ & $160-160$ & $158-160$ & & & & & & \\
\hline DXYS10085 & $212-224$ & $228-224$ & $224-220$ & $224-220$ & $222-216$ & $220-216$ & $216-216$ & & & & & & \\
\hline
\end{tabular}

Abbreviations: LMD, Langer mesomelic dysplasia; LWD, Léri-Weill dyschondrosteosis; SHOX, short stature homeobox gene; $\sim$, haplotype could not be definitely defined but is predicted to be as stated.

The SHOX A170P mutation is located between markers DXYS10092 and DYS290. The common haplotype segregating with the mutation and phenotype is indicated in bold. The precise haplotype associated with the SHOX mutation was determined in 10 of the families, whereas a predicted haplotype was determined in families 7 and 10 , as DNA was only available from the proband.

for the A170P mutation, with that of a 23-week normal fetal growth plate (Figure 3). SHOX was observed in the resting, proliferative and hypertrophic zones of both the control and the LMD growth plate. The chondrocytes appeared to be enlarged and in pairs in the reserve zone of the LMD growth plate. Their columnar stacking in the proliferative zone was also disorganized, with the chondrocytes appearing in less defined columns and in smaller clusters. The controls for antibody-specific staining are shown in Supplementary Figure 2.

\section{DISCUSSION}

A total of 12 families with multiple affected LWD and LMD individuals were found to carry the A170P SHOX mutation in heterozygosity and homozygosity, respectively. A common haplotype around
SHOX was observed in all 12 families, suggesting the presence of a common ancestor. The probands originate from different Spanish provinces, 11 were of gypsy origin, thus suggesting that the common ancestor belonged to this ethnic group. The probability of this SHOX haplotype in the unaffected Spanish Gypsy population was low and even lower in the Spanish population. The haplotype block spanned between 77 and $165 \mathrm{~kb}$. Multiple recombination events had occurred at increased distances. A study of linkage disequilibrium around SHOX showed that in Northern-European populations, disequilibrium decays rapidly with physical distance and that the recombination rate of the PAR 1 is approximately 20 -fold higher than the genome average. $^{21}$ Therefore, it is not unexpected to observe multiple recombinational events in these families. 

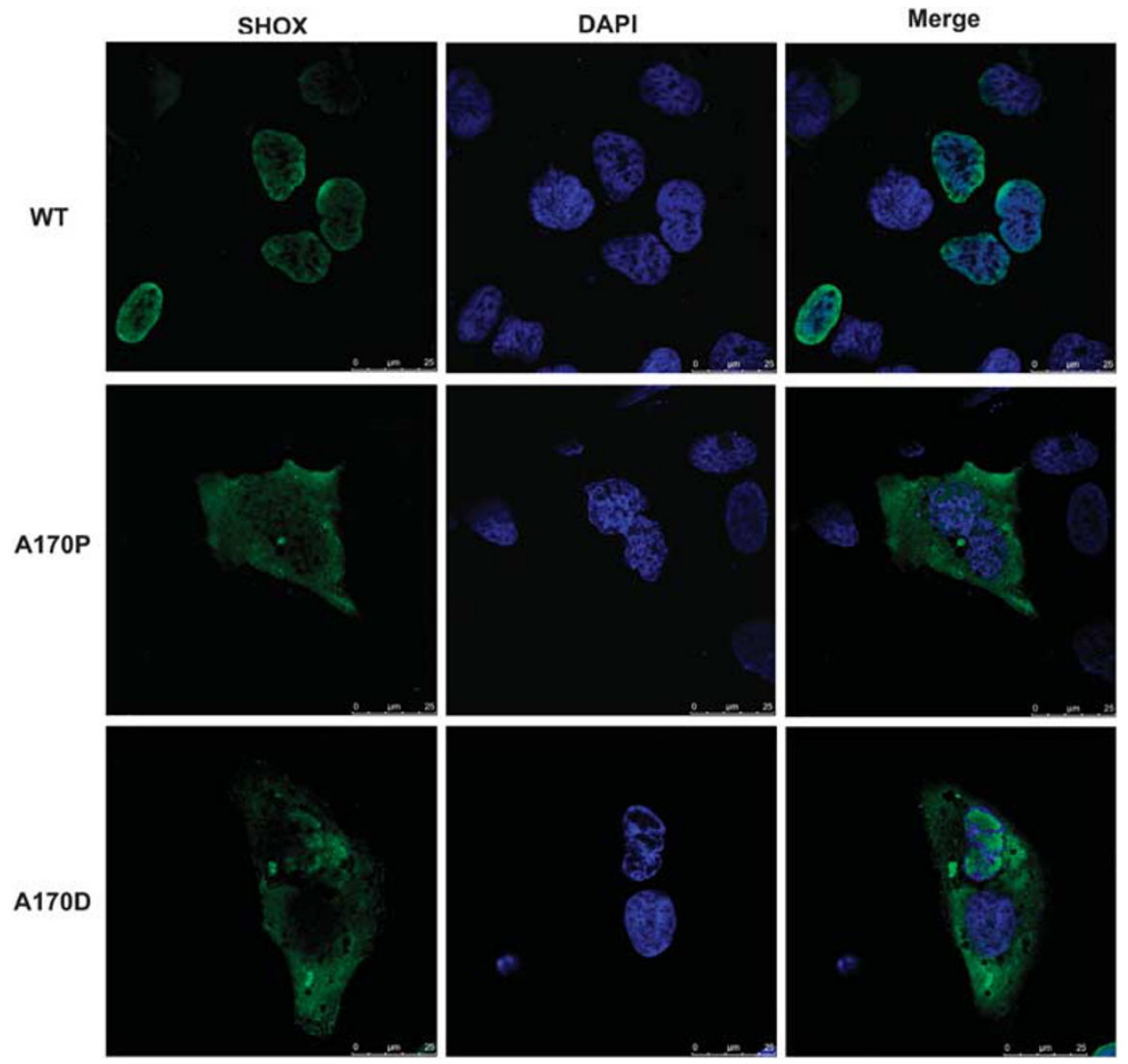

Figure 2 Subcellular localization studies of wild-type and mutant A170P and A170D SHOX proteins. U2OS cells were transiently transfected with the SHOX wild-type and mutant constructs. SHOX expression was detected using a rabbit polyclonal SHOX antibody and the nuclei were counterstained with DAPI. Images were observed using a confocal microscope at $\times 20$ magnification.

As the A170P mutation was identified in 11 Spanish Gypsy families, we investigated its history and incidence of this mutation in the European Gypsy population. A panel of population controls from Bulgaria, representing Gypsy groups that remained in the Balkans during the early Gypsy diaspora across Europe, was screened for the A170P mutation. No mutation was identified, but failure to identify any mutation carrier does not rule out its presence in the Eastern Europe, where it could be confined to specific sub-isolate(s) not represented in the panel or could occur at very low frequency. The A170P SHOX mutation should therefore be the first choice in diagnostic analyses of LWD/LMD patients from this ethnic group. The mutation is more prevalent in the Spanish Gypsy population, in which six out of the seven prenatal diagnoses that we have undertaken in recent years belonged to high-risk Gypsy families.

Interestingly, the A170P mutation was highly penetrant, with all carriers presenting with mesomelic shortening of the limbs and the Madelung deformity, characteristics of LWD, in contrast to other SHOX mutations, which may present as either LWD or idiopathic short stature (ISS, MIM 300582). Only one individual did not fulfill the criteria of short stature (family 12, individual III.1). The high penetrance is likely to be due to the functional importance of alanine 170 , which resides within a five non-classic basic amino acid nuclear localization signal (AKCRK), located in the homeodomain of SHOX. ${ }^{16,18}$ Alanine 170 is one of the four amino acids which are invariably conserved in the homeodomain of all paired related homeodomain proteins. The A170P mutation has been shown to result in the incorrect subcellular localization of the SHOX protein, affecting the translocation from the cytosol to the nucleus, which would lead to the functional impairment of the transcription factor. ${ }^{16}$ Indeed, these amino acids have been shown to not only be essential for SHOX nuclear localization, but may also participate in other processes such as DNA binding, dimerization ${ }^{22}$ and interaction with its cofactors. ${ }^{20}$

We have investigated, for the first time, the effects of the A170P mutation on the growth plate of the radius and ulna of a LMD fetus. The chondrocytes in the reserve zone appeared enlarged and in pairs, whereas the proliferative zone was disorganized, with the columns appearing shortened and stacked side by side rather than in an orderly stacking. Despite this, the pattern of SHOX expression was unchanged between the normal growth plate and that of the LMD fetus. The histology of the growth plate in LMD fetuses has been previously described, ${ }^{23}$ but at this time point, the implicated gene had not been discovered. Later, comparison of the histology and SHOX expression in the growth plate of normal and four LWD fetuses, two with SHOX deletions, one with a splice site mutation and one with a deletion of the SHOX enhancer region was studied. ${ }^{24}$ In all cases of LWD and LMD, disorganization of the chondrocyte columnar stacking has been observed.

We have also identified two independent LWD probands with a previously unreported mutation (c.509C $>$ A) affecting the same amino acid, p.A170D. We expressed the mutant protein in U2OS osteosarcoma cells and showed that the A170D mutant protein, as shown with the A170P mutation, failed to translocate to the nucleus, thus leading to the malfunction of the transcription factor. 

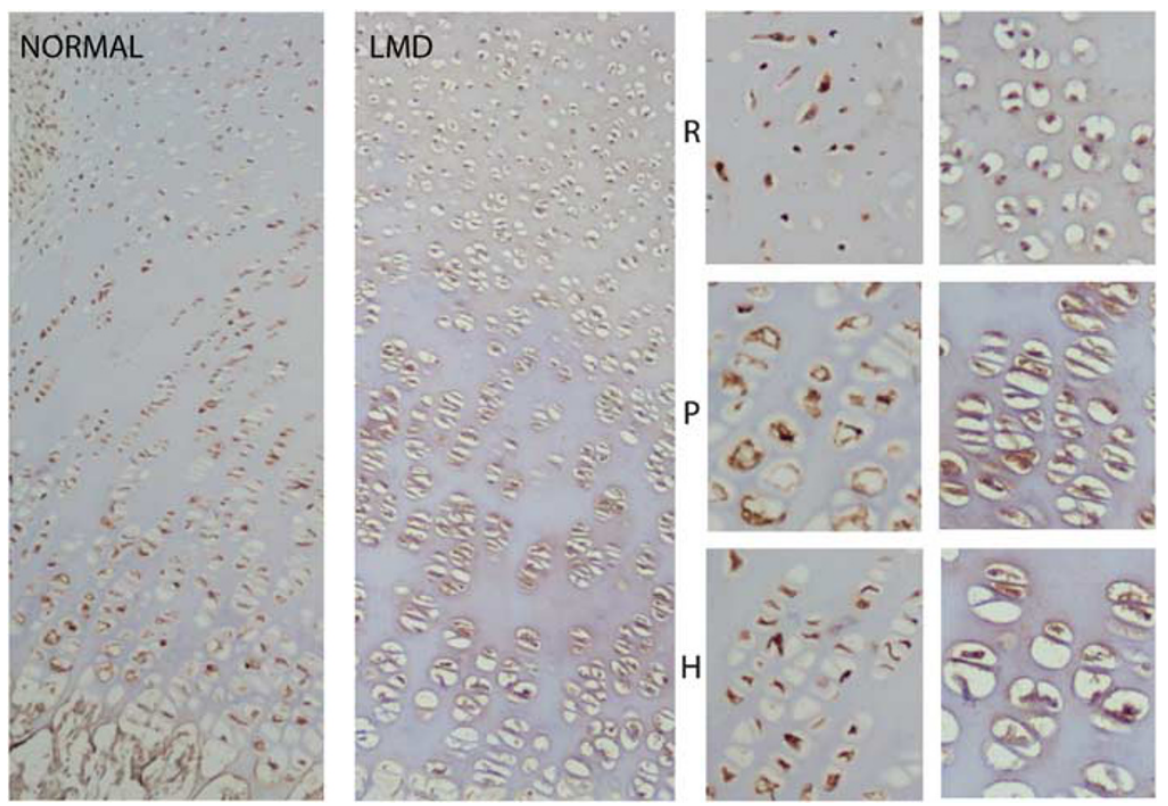

Figure 3 SHOX expression in the human fetal growth plate. Immunohistochemistry performed on the radius and ulna of a 22-week old LMD fetus homozygous for the A170P mutation (family 11, IV.9) and a 23-week old normal fetus. DAB immunostaining ( $\times 10$ magnification) performed with anti-SHOX antibody in the LMD and normal fetal growth plate, showing the reserve $(R)$, proliferative $(P)$ and hypertrophic $(H)$ zones. Specific staining can be observed in all zones of the LMD and control growth plates. The chondrocytes appear enlarged and in pairs in the reserve zone of the LMD growth plate. The chondrocyte columnar stacking in the proliferative zone of the LMD preparations is slightly disorganized compared to the normal growth plate. Controls for antibody specificity are shown in Supplementary Figure 2.

Including the cases reported in this study and identified by our group, six different mutations within the five amino acids of the SHOX nuclear localization signal have now been reported in $28 \mathrm{LWD} /$ LMD/ISS probands (unpublished data and SHOX database: http:// hyg-serv-01.hyg.uni-heidelberg.de/lovd/index.php?select_db=SHOX). Five additional mutations affecting the adjacent amino acids arginine 168 and 169 have been reported in 14 further LWD/ISS cases. Although it has been experimentally shown that these two amino acids are not included within the minimal nuclear localization signal, it has been postulated that they may support the minimal signal by their basicity. ${ }^{18}$ Thus, the nuclear localization signal and adjacent basic amino acids appear to be a frequently mutated region associated with LWD and ISS. A note of caution must be mentioned in the detection of mutations in the nuclear localization signal, as both the A170P and A170D mutations result in a false positive deletion of SHOX exon 4 using the commercial MLPA assay.

In conclusion, we have identified A170P as the first common SHOX mutation in Spanish LWD individuals, which appears to have arisen from a common ancestor, most likely of Gypsy origin. Thus, it is advisable to screen the A170P mutation as a first step in the genetic screening cascade of SHOX/PAR1 alterations in Spanish gypsies with LWD or LMD. Moreover, we have also identified a novel mutation, A170D, altering the same conserved alanine 170 residue, which also impairs the nuclear localization of the protein.

\section{CONFLICT OF INTEREST}

The authors declare no conflict of interest.

\section{ACKNOWLEDGEMENTS}

We thank Noelia Sanchez for her technical help, Charo De Pablo Diaz for donating the Spanish gypsy controls and to all the clinicians involved with these families. This work was supported by grants from the 'Ministerio de Ciencia e
Innovación' (MICINN, SAF2006-00663 and SAF2009-08230). VB-T was supported by a FPI Ph.D fellowship from the MICINN (SAF2006-00663), SB-S by a CIBERER postdoctoral fellowship and MA-C by a postdoctoral fellowship from MICINN (SAF2006-00663).

1 Leri A, Weill J: Une affection congenitale et symetrique du developpement osseux: la dyschondrosteose. Bull Mem Soc Med Hosp 1929; 35: 1491-1494.

2 Langer Jr LO: Mesomelic dwarfism of the hypoplastic ulna, fibula, mandible type. Radiology 1967; 89: 654-660.

3 Rao $\mathrm{E}$, Weiss $\mathrm{B}$, Fukami $\mathrm{M}$ et al: Pseudoautosomal deletions encompassing a novel homeobox gene cause growth failure in idiopathic short stature and Turner syndrome. Nat Genet 1997; 16: 54-63.

4 Ellison JW, Wardak Z, Young MF, Gehron Robey P, Laig-Webster M, Chiong W: PHOG, a candidate gene for involvement in the short stature of Turner syndrome. Hum Mol Genet 1997; 6: 1341-1347.

5 Rao E, Blaschke RJ, Marchini A, Niesler B, Burnett M, Rappold GA: The Léri-Weill and Turner syndrome homeobox gene SHOX encodes a cell-type specific transcriptional activator. Hum Mol Genet 2001; 10: 3083-3091.

6 Shears DJ, Guillen-Navarro E, Sempere-Miralles M et al: Pseudodominant inheritance of Langer mesomelic dysplasia caused by a SHOX homeobox missense mutation. Am J Med Genet 2002; 110: 153-157.

7 Campos-Barros A, Benito-Sanz S, Ross JL, Zinn AR, Heath KE: Compound heterozygosity of SHOX-encompassing and downstream PAR1 deletions results in Langer mesomelic dysplasia (LMD). Am J Med Genet Part A 2007; 143: 933-938.

8 Bertorelli R, Capone L, Ambrosetti F et al: The homozygous deletion of the 3' enhancer of the SHOX gene causes Langer mesomelic dysplasia. Clin Genet 2007; 72 : 490-491.

9 Belin V, Cusin V, Viot G et al: SHOX mutations in dyschondrosteosis (Léri-Weill syndrome). Nat Genet 1998; 19: 67-69.

10 Shears DJ, Vassal HJ, Goodman FR et al: Mutation and deletion of the pseudoautosomal gene SHOX cause Léri-Weill dyschondrosteosis. Nat Genet 1998; 19: 70-73.

11 Benito-Sanz S, Thomas NS, Huber C et al: A novel class of pseudoautosomal region 1 (PAR1) deletions downstream of SHOX is associated with Léri-Weill dyschondrosteosis (LWD). Am J Hum Genet 2005; 77: 533-544.

12 Fukami M, Okuyama T, Yamamori S, Nishimura G, Ogata T: Microdeletion in the SHOX $3^{\prime}$ region associated with skeletal phenotypes of Langer mesomelic dysplasia in a $45, X$ $46, X, r(X)$ infant and Léri-Weill dyschondrosteosis in her 46,XX mother: implication for the SHOX enhancer. Am J Med Genet Part A 2005; 137: 72-76. 
13 Sabherwal N, Bangs F, Roth $\mathrm{R}$ et al: Long-range conserved non-coding SHOX sequences regulated expression in developing chicken limb and are associated with short stature phenotypes in human patients. Hum Mol Genet 2007; 16: 210-222.

14 Chen J, Wildhardt G, Zhong Z et al: Enhancer deletions of the SHOX gene as a frequent cause of short stature: the essential role of a $250 \mathrm{~kb}$ downstream regulatory domain. J Med Genet 2009; 46: 834-839.

15 Benito-Sanz S, Barroso E, Heine-Suñer D et al: Clinical and molecular evaluation of SHOXIPAR1 duplications in Léri-Weill dyschondrosteosis (LWD) and idiopathic short stature (ISS). J Clin Endocrinol Metab 2011; 96: E404-E412.

16 Sabherwal N, Blaschke RJ, Marchini A et al: A novel point mutation A170P in the SHOX gene defines impaired nuclear translcocation as a molecular cause for Léri-Weill dyschondrosteosis and Langer dysplasia. J Med Genet 2004; 41: e83.

17 Sabherwal N, Schneider KU, Blaschke RJ et al: Impairment of SHOX nuclear localization as a case for Léri-Weill syndrome. J Cell Science 2004; 117: 3041-3048.

18 Benito-Sanz S, Gorbenko Del Blanco D, Aza-Carmona M et al: PAR1 deletions downstream of SHOX are the most frequent defect in a Spanish cohort of Léri-Weill dyschondrosteosis (LWD) probands. Hum Mutat 2006a; 27: 1062.
19 Benito-Sanz S, Gorbenko del Blanco D, Huber C et al: Characterization of SHOX deletions in Leri-Weill dyschondrosteosis (LWD) reveals genetic heterogeneity and no recombination hotspots. Am J Hum Genet 2006; 79: 409-414.

20 Aza-Carmona M, Shears DJ, Yuste-Checa P et al: SHOX interacts with the chondrogenic transcription factors SOX5 and SOX6 to activate the aggrecan enhancer. Hum Mol Genet 2011; 20: 1547-1559.

21 May CA, Shone AC, Kalydjieva L, Sajantila A, Jeffreys AJ: Crossover clustering and rapid decay of linkage disequilibrium in the Xp/Yp pseudoautosomal gene SHOX. Nat Genet 2002; 31: 272-275.

22 Schneider KJ, Marchini A, Sabherwal N et al: Alteration of DNA binding, dimerization, and nuclear translocation of SHOX homeodomain mutations identified in idiopathic short stature and Leri-Weill dyschondrosteosis. Hum Mutat 2005; 26: 1-9.

23 Evans MI, Zador IE, Quereshi F, Budev H, Quigg MH, Nadler HL: Ultrasonographic prenatal diagnosis and fetal pathology of Langer mesomelic dwarfism. Am J Med Genet 1998; 31: 915-920.

24 Munns CF, Glass IA, LaBrom R et al: Histopathological analysis of Leri-Weill dyschondrosteosis: disordered growth plate. Hand Surg 2001; 6: 13-23.

Supplementary Information accompanies the paper on European Journal of Human Genetics website (http://www.nature.com/ejhg) 